\title{
Retrotransposons of Arabidopsis thaliana expressed in wild-type plants
}

\author{
Sofya Gvaramiya \\ Department of marker-assisted \\ and genomic selection of plant \\ All-Russia Research Institute of \\ Agricultural Biotechnology \\ Moscow, Russia \\ sofia.gvaramia@gmail.com
}

\author{
Murad Omarov \\ Department of marker-assisted \\ and genomic selection of plant \\ All-Russia Research Institute of \\ Agricultural Biotechnology \\ Moscow, Russia \\ muradok98@gmail.com
}

\author{
Ilya Kirov \\ Department of marker-assisted \\ and genomic selection of plant \\ All-Russia Research Institute of \\ Agricultural Biotechnology \\ Moscow, Russia \\ kirovez@gmail.com
}

\begin{abstract}
Mobile genetic elements are widely spread across the genomes of plants and animals. Of them, retrotransposons occupy substantial part of the genome. They had a huge impact on host genome evolution. However, their current activity in plant cell is thought to be very low on both RNA and cDNA (mobilome) levels because multiple silencing pathways. To get deeper insight into the mechanisms of mobilome formation we carried out identification of transcribed TEs of model plant, Arabidopsis thaliana. Using bioinformatics and 'wet' approaches we found evidence of expression of 6 TEs of A.thaliana. This opens a door for further functional studies of how some TEs can overcome the genome defense against retrotransposon activity.
\end{abstract}

Keywords - retrotransposons, Arabidopsis thaliana, transposable elements, retrotranscriptome, retrotranspososns mobility

Motivation and aim

Motivation

Repetitive elements constitute a major part of higher plant genome [1]. The ubiquitous class of these elements is retrotransposons (TEs) - mobile genetic elements, which move through host genome via "copy-paste" mechanism, including RNA transcription, reverse transcription with formation of cDNA and its insertion. A large number of active retrotransposons are suppressed by various types of methyltransferases like MET1, CMT3, KYP and by RNAdirected DNA methylation (RdDM) [2]. Therefore, it is hard to study biological processes connected with transposable elements. However, while most of the TEs are silenced in the genomes, the recent studies showed that some of them can form RNAs in somatic tissues under non-stressed conditions raising the question of how some TEs can overcome the genome defense mechanisms. A.thaliana being a model plant provides unique set of the tools, but no TEs transcribed in wild-type non-stressed plants have been described so far.

Aims

The aim of our study was to detect retrotransposons expressed in A. thaliana WT (Col-0) and ddm 1 mutant plants under non-stressed conditions.

\section{Methods}

\section{Bioinformatic analysis}

Data analysis of LTR-retrotransposons expression was performed using proper pipeline, combined different programs and designed for this research.

\section{Molecular methods}

DNA was extracted by CTAB method from leaf tissue and exposed polymerase chain reaction.

RNA was isolated from frozen $A$. thaliana plants with Trizol reagent (Evrogen) according to applied protocol. Extracted RNA was treated with DNAse I (Qiagen). After treatment, RNA was subjected RT-PCR through special kit (Biolabmix).

\section{Results}

In our research, we have verified mutant lines of $A$. thaliana by target genes and rated the transcriptional activity of EVD retrotransposon as marker for these plants [3], [4].

We have found six active retrotransposons, which expressed in ddm 1 and wild type plants of Arabidopsis by means of our bioinformatic approach and RT-PCR analysis. To confirm their mobility the presence of extrachromosomal circular DNA was analysed. The results of this work provide unique cases for future studying of retrotransposone evolution and functional activity.

\section{Acknowledgment}

The work was carried out with the financial support of The Russian Foundation for Basic Research (RFBR grant № 2034-70032).

\section{References}

[1] Slotkin R. K., Martienssen R. A. (2008) Transposable elements and the epigenetic regulation og the genome. Nature Rev. Genet. 8: 272-285.

[2] Oberlin S., Sarazin A., Chevalier C., Voinnet O., Mari-Ordonez A. (2017) A genome-wide transcriptome and translatome analysis of Arabidopsis transposons identifies a unique and conserved genome expression strategy fot Ty1/Copia retroelements. Genome Res. 27: 1549-1562.

[3] Reinders J., Mirouze M., Nicolet J., Paszkowski J. (2009) Parent-oforigin control of transgenerational retrotransposon proliferation in Arabidopsis. EMBO reports. 14(9): 823-828.

[4] Tsukahara S., Kobayashi A., Kawabe A., Mathieu O., Miura A., Kakutani T. (2009) Bursts of retrotransposition reproduced in Arabidopsis. Nature. 461: 423-426. 